\title{
Epididymal tuberculosis: Case report
}

J. Shavadia, MBChB and A. Abdallah, MBChB, H Dip Surg (SA), FRCS, Aga Khan University Hospital, Nairobi, Department of Surgery, P.O. Box 30270, 00100, Nairobi, Kenya

Correspondence and reprint requests to:

Dr. A. Abdallah, Aga Khan University Hospital, Nairobi, Department of Surgery, P.O. Box 30270, 00100, Nairobi, Kenya, Email: abdulkarim.abdallah@aku.edu

\section{Summary}

The increasing incidence of extrapulmonary tuberculosis (EPT) parallels the global increase in the prevalence of tuberculosis. Epididymal tuberculosis (TB), arareform of EPT, may present both diagnostic and treatment challenges.

A case of epididymal TB where the diagnosis was made after an orchidectomy for a suspected malignancy is discussed. The thrust of this presentation is to highlight the varied clinical manifestations of tuberculosis, the great masquerader, in our local setting. The case highlights the need to have a high level of diagnostic suspicion. This will limit unnecessary surgical procedures and prevent complications associated with delay in initiating treatment.

\section{Introduction}

Genitourinary tuberculosis (GUTB) is relatively rare despite the high and rising incidence and prevalence of all forms of tuberculosis in Africa (1). In a survey conducted by Kennedy et al, GUTB accounted for $27 \%$ of extrapulmonary TB, with the kidney accounting for most infections (2). Where the genital organs are involved, the epididymis is the most affected. Dissemination is thought to occur either by downward extension of a renal focus or hematogenously: directly to the epididymis or indirectly via retrograde extension from a hematogenously seeded prostate (3-5).

Diagnosing epididymal TB can be challenging and an important step to elucidate this diagnosis is from the patient's history. Prior TB infection and immunocompromised states in the medical history are important considerations; although lengthy latency periods between pulmonary $\mathrm{TB}$ and the appearance of genitourinary $\mathrm{TB}$ have been reported.

We report a case of epididymal TB in a previously well 39-year-old man that presented as a left testicular mass.

\section{Case report}

A 39 year old previously healthy businessman presented with a one week history of left testicular pain and swelling. This was preceded by a three week history of scrotal discomfort. He did not give history of trauma to the groin or any history of fever, urethral discharge or treatment for tuberculosis. The past medical history was unremarkable, and he was sexually active with a single partner.

On physical examination, he was afebrile and had stable vital signs. Scrotal examination revealed a moderately tender left extratesticular mass, approximately $1 \mathrm{~cm}$ in size, with no associated inguinal lymphadenopathy. The rest of his physical examination was non contributory.

A scrotal ultrasound scan confirmed a well circumscribed, unilateral, round, isoechoic solitary lesion measuring $1.1 \mathrm{~cm} \times 0.98 \mathrm{~cm}$ in continuity with a thickened epididymis (Figure 1). Both testes including on color Doppler flows were reported normal.

Though a benign condition was the most likely diagnosis, a primary epididydimal malignancy was considered preoperatively. He subsequently underwent exploration which 
revealed a thick, hard inflamed epididymis in its whole length. Histopathological sections of the epididymis showed heavy infiltration by mixed inflammatory cells forming ill defined granulomas (Figures 2). Ziehl Nielsen stain on the affected sections revealed acid alcohol fast bacilli (Figures 3). The patient was started on a four-drug based antituberculous regime. On follow-up five months later, he was reported to be asymptomatic.

Figure 1: Round lesion in continuity with a thickened epididymis on scrotal scan



Figure 2: Granuloma on epididymal specimen



Figure 3: Acid alcohol fast bacilli on epididymis



\section{Discussion}

Genitourinary tuberculosis presents a diagnostic challenge. It occurs in both HIVinfected and non infected patients (6-10). Other important considerations include previous exposure to $\mathrm{TB}$, elucidation of other forms of immunodeficiency such as post chemotherapy and post transplant states and exposure to bacille-Calmette-Guerin therapy for treatment of urinary bladder cancer (11).

Commonly, patients present with scrotal/ testicular mass with or without pain/tenderness $(6,12)$. Symptoms are usually insidious and progressive, and can clinically be confused with other infections, cysts and tumors. The index patient presented with a three week history of scrotal discomfort, and on exploration thought to have an epididymal malignancy necessitating an orchidectomy. He however did not have a pre-operative chest radiograph which would have been important since a preoperative diagnosis of malignancy was being considered. It would also have consolidated evidence for extra-pulmonary tuberculosis if a primary lung focus was identified. The patient also had sonographically normal testis, making the diagnosis of testicular malignancy less likely. For this reason, the patient did not have any pre-operative testicular tumor markers performed.

Since primary malignant tumors of the epididymis are extremely rare, a high index of suspicion is required to make a diagnosis of epidydimal TB especially in a patient from a TB endemic zone, as in the index case, who presented with a painful scrotal mass and sonographically normal testes. Other presentations of genitourinary $\mathrm{TB}$ that have been reported include fever and dysuria which were absent in this patient. Others like scrotal abscess and scrotal sinus formation probably occur late $(6,9,12)$. Although unilateral testicular involvement occurs more commonly, bilateral involvement has been described (12).

Since a definitive diagnosis can only be made on histologic examination and culture of the affected specimen, tools such as frozen section analysis (FSA) (13) are increasingly being used for testicular preservation. In the index patient, 
a FSA would have been beneficial and may have led to a testicular sparing surgery. Fine needle aspirate cytology has also been successful in making a diagnosis of GUTB $(14,15)$. This may not have been useful in our patient due to the potential risk of tumor seeding of a suspected malignancy. However, in situations where the index of suspicion for tuberculosis is high, Fine Needle Aspirate for Cytology (FNA) would be helpful in preventing an unnecessary orchidectomy to establish a diagnosis. Polymerase chain reaction (PCR) of urine has also been reported to be of useful diagnostic yield with a sensitivity and specificity of $95.59 \%$ and $98.12 \%$ respectively (16).

The recommended therapy for patients with GUTB in our country is chemotherapy with the standard four-drug regime (rifampicin, isoniazid, pyrazinamide and ethambutol) for two months followed by two drug regime (rifampicin and isoniazid) for the next four months (17). This is in keeping with work done by Gow who demonstrated that all cases of genitourinary TB will respond to a short course of anti-tuberculous therapy, which need not be longer than six months (18). Rajpal et al however used intermittent thrice weekly therapy under direct supervision to successfully treat a patient with GUTB (7). Surgical intervention however becomes necessary in patients with scrotal abscesses and in patients who have extensive disease that is resistant to chemotherapy (19).

This case illustrates the variable presentation of tuberculosis and the high clinical index required to clinch a diagnosis of genitourinary TB. An important lesson arising from this case is that in patients presenting with an epidydimal mass, a thorough history, physical examination and fine needle aspiration prior to surgical exploration may translate into better patient management and prevent unnecessary orchidectomies especially in our setting where the underlying prevalence of tuberculosis is high.

\section{References}

1. Published in Global TB Control 2007.

2. Kennedy D.H. Extrapulmonary tuberculosis. In: Ratledge C., Stanford J.L. and Grange J.M. eds. The biology of the mycobacteria. Vol. III. New York: Academic Press. 1989; 244-284.

3. Porter M.P., Eubank W.B. and Krieger J.N. Genitourinary tuberculosis a focused update for the practicing urologist. Contemp. Urol. 2001; 13: 34-48.

4. Petersen L., Mommsen S. and Pallisgaard G. Male genitourinary tuberculosis report of 12 cases and review of the literature. Scand. J. Urol. Nephrol. 1993; 27: 425-428.

5. Chung J., Kim M., Lee T., et al. Sonographic findings in tuberculous epididymitis and epididymo-orchitis. J. Clin. Ultrasound. 1997; 25: 390-394.

6. Orakwe J.C. and Okafor P.I. Genitourinary tuberculosis in Nigeria; a review of thirty-one cases. Niger J. Clin. Pract. 2005; 8: 69-73.

7. Rajpal S., Dhingra V.K., Malik M., et al. Tuberculous epididymo-orchitis treated with intermittent therapy: A case report. Indian J. Allergy Asthma Immunol. 2002; 16: $51-54$.

8. Demond N., Lynch M., Murphy D., et al. Tuberculous epididymitis: A case report in an HIV seropositive male. Int. J. STD AIDS. 1993; 4: 178-179.

9. Goodman P., Maklad N.F., Verani R.R., et al. Tuberculous abscess of the testicle in AIDS: Sonographic demonstration. Urol. Radiol. 1990; 12: 53-55.

10. Bannur H.B., Malur P.R. and Dhorigol V.M. Tubercular orchitis in a patient with AIDS: report of a case with fine needle aspiration diagnosis. Acta. Cytol. 2007; 51: 459-60.

11. Muttarak M., Lojanapiwat B., Chaiwun B., et al. Preoperative diagnosis of bilateral tuberculous epididymo-orchitis following intravesical bacillus Calmette-Guérin therapy for superficial bladder carcinoma. Australia's Radiol. 2002; 46: 183-185.

12. Viswaroop B.S., Kekre N. and Gopalakrishnan G. Isolated tuberculous epididymitis: A review of forty cases. J. Postgrad. Med. 2005; 51: 109-111.

13. Connolly S.S., D' Arcy F.T., Bredin H.C., et al. Value of frozen section analysis with suspected testicular malignancy. Urology. 2006; 67: 162-165. 
14. Wolf J.S. and McAninch J.W. Tuberculous epididymoorchitis: Diagnosis by fine needle aspiration. J. Urol. 1991; 145: 836-838.

15. Kumar P.V., Owji S.M. and Khezri A.A. Tuberculous orchitis diagnosed by fine needle aspiration cytology. Acta. Cytol. 1996; 40: 1253-1256.

16. Moussa O.M., Eraky I., El-Far M.A., et al. Rapid diagnosis of genitourinary tuberculosis by polymerase chain reaction and non-radioactive DNA hybridization. J. Urol. 2000; 164: 584-588.
17. International Standards for Tuberculosis Care (ISTC) Jan 2006.

18. Gow J.G. Genitourinary tuberculosis: a 7-year review. Brit. J. Urol. 1979; 51: 239-244.

19. Carl P. and Stark L. Indications for surgical management of genitourinary tuberculosis. World J. Surg. 1997; 21: 505-510. 